\title{
A Comparative Study of Channel Estimation Techniques for OFDM in DVB-T2
}

\author{
Ahmed H.Eldieb \\ Electronics and \\ Communications Eng. Dept., \\ Higher Institute of Engineering, \\ Modern Academy \\ Cairo, Egypt
}

\author{
Mona Z.Saleh \\ Electronics and \\ Communications Eng. Dept., \\ Faculty of Engineering, \\ Ain Shams University \\ Cairo, Egypt
}

\author{
Salwa Elramly \\ Electronics and \\ Communications Eng. Dept., \\ Faculty of Engineering, \\ Ain Shams University \\ Cairo, Egypt
}

\begin{abstract}
Orthogonal Frequency Division Multiplexing (OFDM) with pilot symbol assisted channel estimation is a well-known multicarrier technique for high rate transmission over wireless frequency selective fading channels. In this paper a comparative study of channel estimation techniques is presented based on a pilot arrangement used in the second generation for Digital Video Broadcasting Terrestrial (DVBT2) system in time-varying frequency-selective fading channels. A modified method based on Domain Transform Least Square Estimation (DTLSE) has been proposed which reduces the computational complexity by avoiding the use of the matrix inversion .The estimation matrix size for obtaining Channel Impulse Response (CIR) depends only on the length of the channel not on the number of pilot subcarriers nor the size of OFDM symbol. Two Dimensional Linear Interpolation (2-DLI) is discussed which performs better than one Dimensional Linear Interpolation (1-DLI)but suffers from long processing delay and higher sensitivity to Doppler frequency shifts. The Minimum Mean Squared Error (MMSE) channel estimation is also considered as a reference method because it has a good performance close to the perfect estimation at the cost ofhigh computational complexity and the necessity of prior knowledge ofchannel statistics. The proposed method is compared with three other methods DTLSE, 2-DLI and MMSE.The proposed method provesto give a better performance compared to the other methods which suffer from either a much higher computational load or less robustness to Doppler shifts.
\end{abstract}

\section{General Terms}

Channel estimation, wireless systems.

\section{Keywords}

Digital video broadcasting system for terrestrial second generation (DVB-T2), domain transform least square estimation (DTLSE),two dimensional linear interpolation (2DLI),minimum mean square estimation (MMSE).

\section{INTRODUCTION}

OFDM provides an effective and low complexity means of reducing intersymbol interference for transmissions over frequency-selective fading channels. It has been widely applied in wireless communication systems due to its high bandwidth efficiency, high transmission capability and its robustness against multipath delay [1]. OFDM has been adopted by several wireless systems and standards such as WLAN IEEE802.11a/n, 4G LTE, WiMAX IEEE802.16d/e, Digital Audio Broadcasting (DAB), Terrestrial Digital Video Broadcasting DVB-T and DVB-T2 [2].
At the OFDM system transmitter side, the binary data are initially grouped and mapped to signal constellation points, such as QPSK or 16-QAM. Then, the pilots are inserted either as a comb type, block type or a compromise of both (e.g. scattered pilots). The data sequence is then transformed into time domain through IFFT. A guard interval is inserted to prevent inter-symbol interference. The signal is finally applied to a DAC for transmission on channel [3].

Channeleffect, which is caused by the channel characteristics such as attenuation distortion, delay distortion and noise, is compensated by channel estimation at the receiver side. Channel estimation is usually performed by transmitting training pilot symbols on sub-carriers these pilots are known to the receiver. The system performance and computational complexity are twoimportant requirements for selecting the optimum technique of the channel estimation [4].

Channel estimation in frequency-domain pilot assistedOFDM Systems can be obtained by employing theleast Squares (LS)estimate at pilot sub-channels position. Then, the complete Channel Transfer Function (CTF) at allsubcarriers are calculated from interpolation either in frequency-direction (1-D) only or both time and frequency directions (2-D).The (2-D) performs better than (1-D) interpolation at the cost of higher sensitivity to Doppler frequency shifts and long processing delay [5].

The MMSE estimation [6] offers the best Bit Error Rate(BER) but has heavy computational load and needs knowledge of channel statistics. The least square estimation at pilot sub channels can also be employed to estimate time-domain CIR and then transform it to CTF, which is known as the DTLSE [7].

The proposed estimation method needs a single OFDM symbol for getting channel impulse response instead of multiple ones. It also works for any frequency pilot pattern without needing prior knowledge of channel statistics while in the same time considerably reducing the computational load through avoiding the usage of matrix inversion. It is also worth mentioning that the size of the estimation matrix for obtaining CIR depends only on the length of the channel not the number of pilot subcarriers or the size of OFDM symbol.

The paper is organized as follows; the system description and principle of channel estimation are explained in section (2); performance analysis of the modified method compared with other methods is presented in section (3); computational load of the considered methods are calculated in section (4); thesimulation results are presented in section (5) while section (6) concludes the paper. 


\section{SYSTEM DESCRIPTION}

Within a typical OFDM system receiver, after removing the guard interval, demodulation is performed by FFT. When the length of guard interval is longer than the length of channel impulse response, ISI could be eliminated. In this case channel can be viewed as a set of parallel subchannels and the received signal isrepresented as

$$
\mathrm{Y}_{k}=\mathrm{H}_{k} \mathrm{X}_{k}+\mathrm{W}_{k} k=0, \ldots, \mathrm{K}-1
$$

where $Y_{k}$ represents the received signal in complex baseband, $\mathrm{X}_{\mathrm{k}}$ denotes the transmitted signal, $\mathrm{H}_{\mathrm{k}}$ and $\mathrm{W}_{\mathrm{k}}$ are the Channel Frequency Response (CFR) and the additive white Gaussian noise, respectively, $\mathrm{K}$ is the total number of subcarriers and $\mathrm{k}$ is the index of subcarrier within an OFDM symbol [8], [9].

Channel estimation with the aid of pilots in OFDM systems is performed by estimating the CFR at the pilot locations. This is obtained by comparing the received pilot subcarriers with the transmitted one which are known to the receiver. Let $K_{P}$ be the total number of pilots and $k_{P}$ denote the index of the pilot subcarrier,the estimation of CFR at a pilot position can be obtained by the LS method as

$$
\widehat{\mathrm{H}}_{k_{P}}=\frac{Y_{k_{P}}}{\mathrm{X}_{k_{P}}}=\mathrm{H}_{k_{P}}+\frac{\mathrm{W}_{k_{P}}}{\mathrm{X}_{k_{P}}}
$$

where $Y_{k_{P}}$ denotes the received pilot subcarrier, $X_{k_{p}}$ is the transmitted pilot subcarrier, and, $\widehat{\mathrm{H}}_{\mathrm{k}_{\mathrm{p}}}$ is the estimation of CFR at the pilot positions. Then, the CFR values at the rest of the subcarriers are calculated from the estimated ones via interpolation [10].In DVB-T2 system for pilot pattern (PP1), the scattered pilots used for channel estimation are spread in both time and frequency directions. As shown in Figure 1, there is one pilot subcarrier out of three subcarriers in the frequency directionand one pilot out of four symbols in time direction. Thus the pilot density for this pilot pattern is $D_{x}=3$ and $D_{y}=4$ plus edge pilots [11].

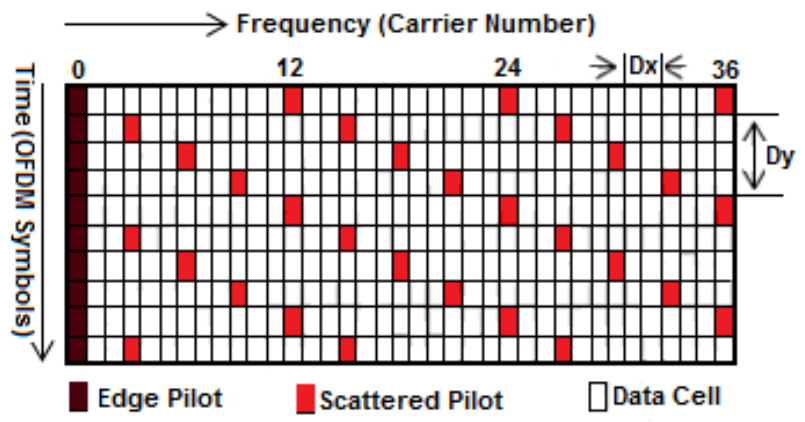

Fig 1: Scattered pilot pattern (PP1) [2].

In the following section the performance analysis of a modified method compared with other methods will be introduced.

\section{PERFORMANCE ANALYSIS OF THE CONSIDERED METHODS}

In this section, four channel estimation methods are presented. Three methods arepreviously used while the fourth is a new proposed algorithm which is less complex than the others.

\subsection{2-DLIEstimation Method}

In this method [5], the CFR at the pilot positions are calculatedat the $k_{p}^{\text {th }}$ pilotsubcarrier and $n_{P}^{\text {th }}$ OFDM symbol as

$$
\widehat{\mathrm{H}}_{\mathrm{n}_{\mathrm{p}}, \mathrm{k}_{\mathrm{p}}}=\frac{\mathrm{Y}_{\mathrm{n}_{\mathrm{P}}, \mathrm{k}_{\mathrm{P}}}}{\mathrm{X}_{\mathrm{n}_{\mathrm{P}}, \mathrm{k}_{\mathrm{P}}}}=\mathrm{H}_{\mathrm{n}_{\mathrm{p}}, \mathrm{k}_{\mathrm{p}}}+\frac{\mathrm{W}_{\mathrm{n}_{\mathrm{P}}, \mathrm{k}_{\mathrm{p}}}}{\mathrm{X}_{\mathrm{n}_{\mathrm{P}}, \mathrm{k}_{\mathrm{P}}}}(3)
$$

The CFR values $\left(\widehat{\mathrm{H}}_{\mathrm{n}, \mathrm{k}}\right)$ at the rest of the subcarriers are calculated from the estimation via interpolation which is applied in both frequency direction (across subcarriers) and time direction (across successive OFDM symbols).At first, interpolation has to be performed in time direction then interpolation is performed in frequency direction to obtain channel coefficients for the remaining data subcarriers. The most common interpolation method is the linear Interpolation (LI) because of its simplicity and low computational complexity. After interpolation of channel coefficients at all data subcarriers the complex received signal is equalized as

$$
\hat{X}_{n, k}=\frac{Y_{n, k}}{\widehat{H}_{n, k}}
$$

where $\mathrm{Y}_{n, k}$ is the complex received signal FFT outputs, $\widehat{\mathrm{H}}_{n, k}$ denotes the estimated and interpolated CFR at all indices and $\widehat{\mathrm{X}}_{n, k}$ is the estimated transmitted signal. This method isconsidered a 2-DLI which suffers from long processing delay andmore sensitivity to Doppler frequency shifts [5].

\subsection{The MMSE Estimation Method}

The MMSE estimation [6], provides the best BER performance. It estimates CTF $\widehat{\mathrm{H}}_{\text {mmse }}$ as

$$
\widehat{\mathrm{H}}_{\mathrm{mmse}}=\mathrm{R}_{k, k_{p}} \cdot \mathrm{R}_{k_{p}, k_{p}}^{-1 / 2} \cdot \mathrm{R}_{k_{p}, k_{p}}^{-1 / 2} \cdot \widehat{\mathrm{H}}_{k_{p}, \mathrm{ls}}
$$

where $\mathrm{R}_{k, k_{P}}$ of size $K \times K_{P}$ is the correlation matrix of complete CTF with pilot subchannels, and $\mathrm{R}_{k_{P}, k_{P}}$ of size $K_{P} \times K_{P}$ is the correlation matrix of pilot subchannels with its LS estimate $\widehat{\mathrm{H}}_{k_{p}, \mathrm{ls}}$. This method suffers from heavy computational load and needsPrior knowledge of channel statistics.

\subsection{DTLSE Estimation Method}

In this method [7], the least square estimation of pilot subchannels is employed to estimate time-domain channel impulse response CIR and then transform it to CTF. The CIR is then given as [7]:

$$
\widehat{\mathrm{h}}_{\mathrm{k}_{\mathrm{p}}}=\left(\mathrm{F}_{k_{P}, k_{P}}^{\mathrm{H}} \mathrm{F}_{k_{P}, k_{P}}+\alpha \mathrm{I}_{k_{P}, k_{P}}\right)^{-1} \cdot \mathrm{F}_{k_{P}, k_{P}}^{\mathrm{H}} \cdot \widehat{\mathrm{H}}_{k_{P}}
$$

where $\mathrm{F}_{k_{P}, k_{P}}$ is a $K_{P} \times K_{P}$ Fourier transform coefficientsmatrix, (. $)^{\mathrm{H}}$ denotes Hermitian transpose, $\mathrm{I}$ is the $K_{P} \times K_{P}$ identity matrix and $\alpha$ is a regularization parameter [12].

The whole effective CTF is estimated by applying N-point FFT on the zero padded estimated effective CIR. In this method the CIR depends only on the number of pilot subcarriers, uses one OFDM symbol, works for any frequency pilot pattern and does not require any knowledge of channel statistics but still use the inverse matrices.

\subsection{The proposed Method}

In this proposedmethod, the use of inverse matrices is avoided as will be shown:

Based on (2) the received pilot subcarriers can be defined as

$$
\mathrm{Y}_{k_{P}}=\widehat{\mathrm{H}}_{k_{P}} \cdot \mathrm{X}_{k_{P}}(7)
$$




$$
=\mathrm{D}\left(\mathrm{X}_{k_{P}}\right) \cdot F_{k_{P}, \mathrm{~L}} \cdot \hat{h}_{L}
$$

where $\mathrm{D}\left(\mathrm{X}_{k_{P}}\right)$ is a diagonal matrix with $\mathrm{X}_{k_{P}}$ on its diagonal, $\hat{h}_{L}$ is the CIR , $F_{k_{P}, \mathrm{~L}}$ is the fast Fourier transform matrix of size $K_{P} \times L$ and $\mathrm{L}$ is the length of the channel. Thenby using the least square solution the $\operatorname{CIR} \hat{h}_{L}$ is obtained as:

$$
\begin{aligned}
\hat{h}_{L}= & \left(\left(\mathrm{D}\left(\mathrm{X}_{k_{P}}\right) \cdot F_{k_{P}, \mathrm{~L}}\right)^{\mathrm{H}} \cdot \mathrm{D}\left(\mathrm{X}_{k_{P}}\right) \cdot F_{k_{P}, \mathrm{~L}}\right)^{-1} \cdot\left(\mathrm{D}\left(\mathrm{X}_{k_{P}}\right) \cdot F_{k_{P}, \mathrm{~L}}\right)^{\mathrm{H}} \\
& . \mathrm{Y}_{k_{P}}(9) \\
\hat{h}_{L} & =\left(\mathrm{F}_{k_{P}, \mathrm{~L}}^{\mathrm{H}} \cdot \mathrm{D}\left(\mathrm{X}_{k_{P}}\right)^{\mathrm{H}} \cdot \mathrm{D}\left(\mathrm{X}_{k_{P}}\right) \cdot F_{k_{P}, \mathrm{~L}}\right)^{-1} \cdot\left(\mathrm{D}\left(\mathrm{X}_{k_{P}}\right) \cdot F_{k_{P}, \mathrm{~L}}\right)^{\mathrm{H}} \\
& . \mathrm{Y}_{k_{P}}
\end{aligned}
$$

Using the fact that $\mathrm{D}\left(\mathrm{X}_{k_{P}}\right)^{\mathrm{H}} \cdot \mathrm{D}\left(\mathrm{X}_{k_{P}}\right)=\mathrm{I}_{k_{P}}$, where $\mathrm{I}_{k_{P}}$ is the identity matrix of size $K_{P} \times K_{P}$

$$
\hat{h}_{L}=\left(\mathrm{F}_{k_{P}, \mathrm{~L}}^{\mathrm{H}} \cdot F_{k_{P}, \mathrm{~L}}\right)^{-1} \cdot\left(\mathrm{D}\left(\mathrm{X}_{k_{P}}\right) \cdot F_{k_{P}, \mathrm{~L}}\right)^{\mathrm{H}} \cdot \mathrm{Y}_{k_{P}}
$$

$\mathrm{F}_{k_{P}, \mathrm{~L}}^{\mathrm{H}} \cdot F_{k_{P}, \mathrm{~L}}=K_{P} \cdot I_{L}$, where $I_{L}$ is the identity matrix of size $\mathrm{L} \times$ Lso that $\mathrm{h}_{\mathrm{L}}$ is given as

$$
\hat{h}_{L}=\left(\frac{1}{k_{P}}\right) \mathrm{I}_{\mathrm{L}} \cdot \mathrm{F}_{k_{P}, \mathrm{~L}}^{\mathrm{H}} \cdot \mathrm{D}\left(\mathrm{X}_{k_{P}}\right)^{\mathrm{H}} \cdot \mathrm{Y}_{k_{P}}
$$

Based on (12) the size of the estimation matrix $\hat{h}_{L}$ for obtaining CIR only depends on the length of the channel and the use of inverse matrices is avoided.

\section{COMPUTATIONAL LOAD OF THE CONSIDERED METHODS}

The computational load or the amount of arithmetic operations is an important factor for distinguishing between the methods of channel estimation.In Strassen algorithm [13] the multiplication betweenann $n_{1} \times \mathrm{n}_{2}$ matrix and $\operatorname{ann}_{2} \times \mathrm{n}_{3}$ matrix requires about $2 \mathrm{n}_{1} \mathrm{n}_{2} \mathrm{n}_{3}$ arithmetic operations and the Inverse of an $\mathrm{n} \times \mathrm{n}$ matrix requires about $5.5 \mathrm{n}^{2.8}$ arithmetic operations. The number of subcarriers is denoted by $\mathrm{N}, \mathrm{N}_{\mathrm{p}}$ is the number of pilots, the ratio of them is $\rho=N_{p} / N$ and $L$ is the length of channel. The 2-DLI computational load is not considered here, because it depends on linear interpolations only which is less than the matrix based methods in complexity.

1) For MMSE, (5), requires [6]: $4 \rho^{2} \mathrm{~N}^{3}+5.5 \rho^{2.8} \mathrm{~N}^{2.8}+$ $3 \rho \mathrm{N}^{2}$ arithmetic operations.

2)For DTLS, (6), requires[7]: $2 \rho^{3} \mathrm{~N}^{3}+5.5 \rho^{2.8} \mathrm{~N}^{2.8}+$ $2 \rho \mathrm{N}^{2}+\rho \mathrm{N}$ arithmetic operations.

3) Forthe proposed Method, (12) requires: $2 \mathrm{~L} \rho^{2} \mathrm{~N}^{2}+$ 2L $\rho$ Narithmetic operations.

From all the previousarithmetic operations, the saving on computational load by the proposed method will be more significant than theothers. Thecomparison between the proposed and other methods in computational load is shown in Figure 2.

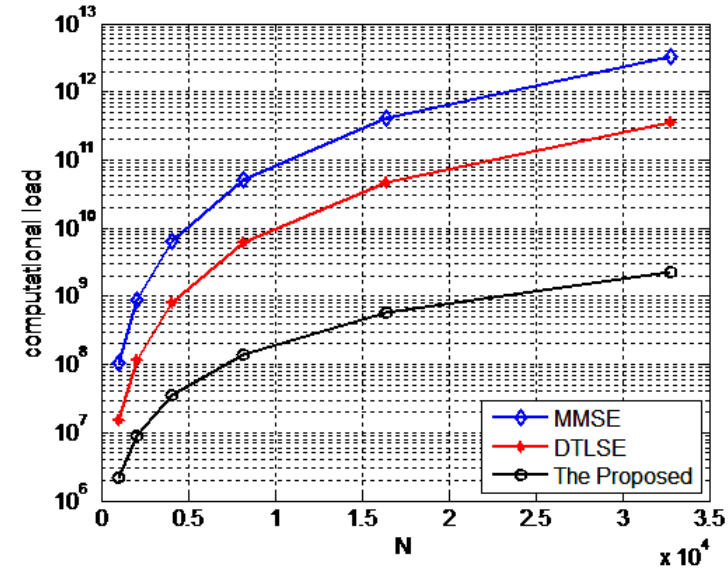

Fig 2: The computational loadfor the considered methods

\section{SIMULATION RESULTS}

The simulated OFDM system is adopted from DVB-T2 [2], with a bandwidth of $8 \mathrm{MHz}$.There are 1024 sub-carriers, Data subcarriers are modulated by quadratic phase-shift keying constellation, Pilot density is $D_{x}=3$ and $D_{y}=4$ plus edge pilots with elementary time of $\mathrm{T}=7 / 64 \mu \mathrm{s}$, and the CP length is 64. Two channel models were investigated in order to evaluate andcompare the performance of the proposed andconsidered methods under different channel conditions with frequency selectivity. The performance under a slow and a higher Doppler frequency shift was also considered.

A typical urban channel profile named TU-6 in [14] is considered. It consists of six taps having wide dispersion in delay and relatively strongpower. Each of them follows the Jakes' Doppler spectrum [15]. The model in [7] was applied in which the taps are considered to be spaced by a multiple of T.The first tap is assumed to be of zero delay, and the channel length is $\mathrm{Lc}=47$. Six taps with nonzero power are positioned at $\mathrm{j}=0,2,5,15,21,46$, with powers equal to $(-3,0,-2,-6$, $-8,-10) \mathrm{dB}$, respectively. A Brazil Channel $\mathrm{D}$ in [16] is also considered with six taps with nonzero power are positioned at $\mathrm{j}=0,5,20,29,57,58$, with powers equal to $(-0.1,-3.9,-2.6$,$1.3,0,-2.8) \mathrm{dB}$, respectively and the channel length is $\mathrm{Lc}=59$.

The BER performance of the proposed method was simulated and compared with 2-DLI, DTLSE and MMSE methods under $50-\mathrm{Hz}$ Doppler frequency shift. It provides BER values $2.5 \mathrm{~dB}$ away from that with perfect channel estimation. This difference is negligible for the MMSE method as shown in figure3. For a high Doppler frequency shift of $300 \mathrm{~Hz}$, The BER results are shown in figure4. The proposed, the DTLSE and the MMSE provide similar performance as in the case of low Doppler frequency shift. There is a BER error floor for the 2-D LI method which shows that it is not robust to high Doppler frequency shifts.

In case of a Brazil channel as shown in figure 5 the BER of the proposed and the other methods under $50-\mathrm{Hz}$ is slightly higher than that of in Fig.3. This means that Brazil channel is more frequency selective than TU-6 channel, because the root-mean-square delay spread of the Brazil channel is $3.55 \mu \mathrm{s}$ and that of TU6 is $1.25 \mu \mathrm{s}$. In figure 6 the BER performance of the proposed methods with 2-DLI, DTLSE and MMSE methods under $300-\mathrm{Hz}$ is simulated. The BER of 2-DLI method is much higher than all methods which provide similar performance as in the figure 5 . 


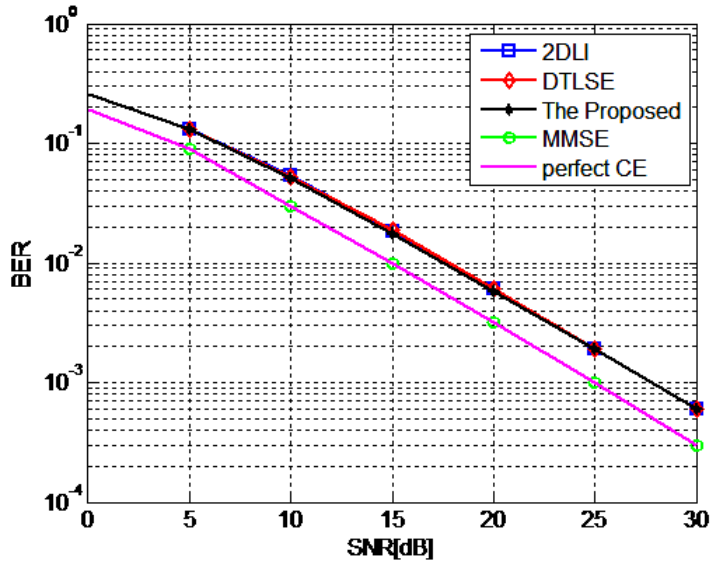

Fig 3: BER vs. SNR for the proposed and other methods in TU-6 for 50-Hz Doppler frequency shift.

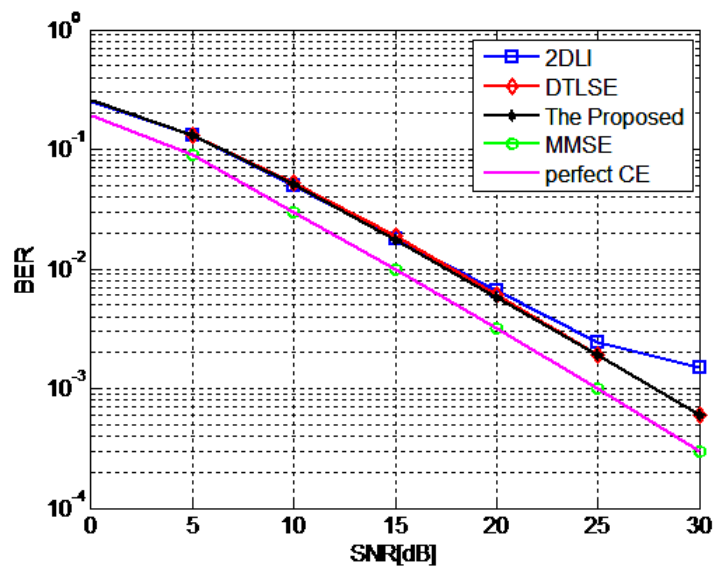

Fig 4: BER vs. SNR for the proposed and other methods in TU-6 for 300-Hz Doppler frequency shift.

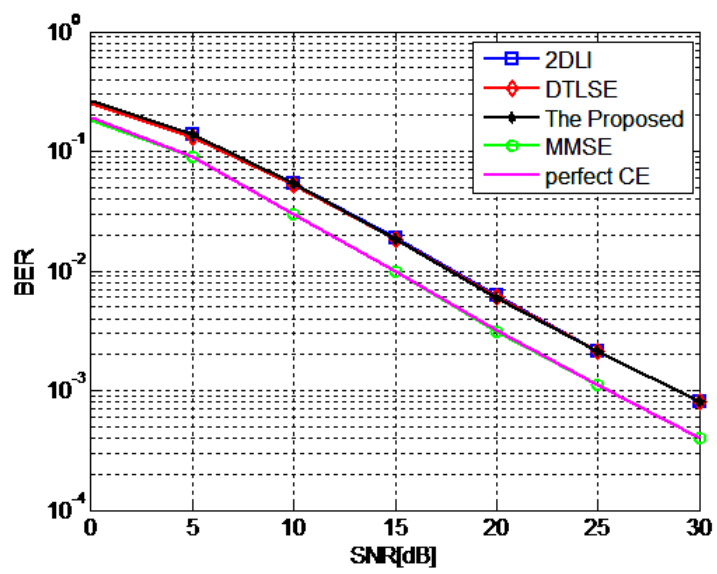

Fig 5: BER vs. SNR for the proposed and other methods in Brazil D for 50-Hz Doppler frequency shift.

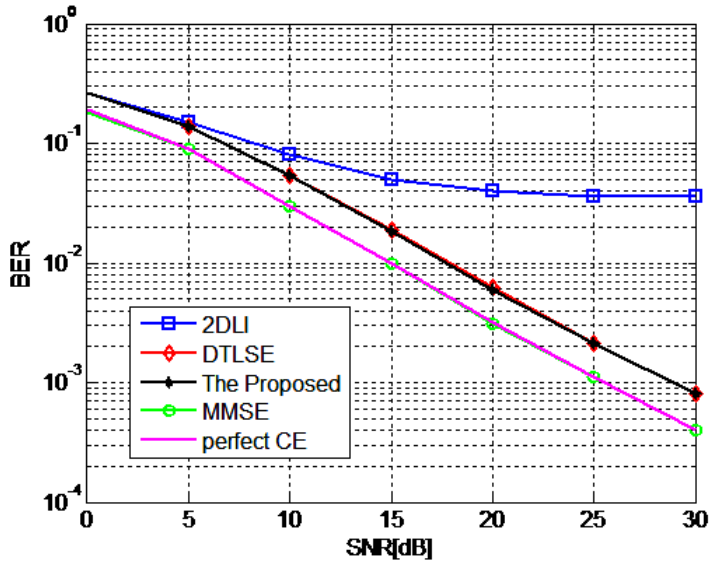

Fig 6: BER vs. SNR for the proposed and other methods in Brazil D. for 300-Hz Doppler frequency shift.

\section{CONCULSION}

A modified method based on DTLSE has been proposed which reduced the computational complexity by avoiding matrix inversion. The size of the estimation matrix for obtaining CIR only depends on the length of the channel not on the number of pilot subcarriers or the size of OFDM symbol.The proposedmethod uses only one OFDM symbol, works for any frequency pilot pattern and does not require any knowledge of channel statistics. The proposed method provides similar channel estimation BER performance as the DTLSE method which still uses the matrix inversion and better than 2-DLI which has less robustness to Doppler frequency shifts. Compared to the reference estimation method MMSE, the proposed method provides BER values $2.5 \mathrm{~dB}$ less than MMSE but the computational load of MMSE compared to the proposed method for a $1 \mathrm{k}$ DVB-T2 mode is 46.7:1 bigger.

\section{RERFERNCES}

[1] R. Prasad, 2004. OFDM for Wireless Communications Systems, Singapore, Artech House.

[2] DVB Document A133, February, 2012. Implementation guidelines for a second generation Digital terrestrial television broadcasting system (DVB-T2).

[3] Sinem Coleri, Mustafa Ergen, Anuj Puri, and Ahmad Bahai, "Channel Estimation Techniques Based on Pilot Arrangement in OFDM Systems," IEEE Transactions on broadcasting, September 2002, Vol. 48, NO. 3.

[4] Yong Soo Cho, Jaekwon Kim,Won Young Yang,ChungGu Kang,"MIMO-OFDM wireless communications with MALAB," John Wiley \& Sons (Asia) Pte Ltd., 2010.

[5] M. Speth, S. Fechtel, G. Fock, and H. Meyr, "Optimum receiver design for OFDM-based broadband tramsmisson-Part II: A case study," IEEETrans. Commun, Apr. 2001, Vol. 49, no. 4, pp. 571-578.

[6] O. Edfors, M. Sandell, J.-J. Van de Beek, S. Wilson, and P. Borjesson, "OFDM channel estimation by singular value decomposition,'JEEE Trans. Commun., Jul. 1998, Vol. 46, No. 7, PP. 931-939. 
[7] Mingchao $\mathrm{Yu}$ and ParastooSadeghi," Study of PilotAssisted OFDM Channel Estimation Methods with Improvements for DVB-T2," IEEE Trans.VehicularTechnology, June 2012, Vol. 61, NO. 5.

[8] D. C. Chang, "Effect and compensation of symbol timing offset in OFDM systems with channel interpolation," IEEE Trans. Broadcast., Dec. 2008, Vol. 54, no. 4, pp. 761-770.

[9] J. Park, J. Kim, M. Park, K. Ko, C. Kang, and D. Hong, "Performance analysis of channel estimation for OFDM systems with residual timing offset," IEEE Trans. Wireless Commun.,July 2006, Vol. 5, no. 7, pp. 16221625 .

[10] Y.S. Lee, H.N. Kim,S. I. Park, and S. I. Lee, "Noise reduction for channel estimation based on pilot-block averaging in DVB-T receivers,"IEEE Trans. Consumer Electron., Feb. 2006,Vol. 52, no. 1, pp. 51-58.
[11] DVB Document A122Frame structure channel coding and modulation for a second generation digital terrestrial television broadcasting system (DVB-T2), June. 2008.

[12] P. C. Hansen, Rank-Deficient and Discrete Ill-Posed Problems.Philadelphia, PA: SIAM, 1998.

[13] T. H. Cormen, C. E. Leiserson, R. L. Rivest, and C. Stein, Introduction to Algorithms. Cambridge, MA: MIT Press, 2001.

[14] DVB Document A133 Implementation guidelines for a second generation digital terrestrial television broadcasting system (DVB-T2), Jun. 2010.

[15] J. G. Prokais, Digital Communications. Singapore: McGraw-Hill, 1995.

[16] Mackenzie, ABERT, SET, "General description of laboratory tests,"DTV Field Test Report in Brazil, July 2000. 